\title{
Overexpression of matrix metalloproteinase-9 in adolescents with primary spontaneous pneumothorax for surgical intervention
}

Chih-Yung Chiu, MD, PhD, ${ }^{\mathrm{a}, \mathrm{c}, \mathrm{d}}$ Tzu-Ping Chen, MD, ${ }^{\mathrm{c}, \mathrm{e}}$ Jim-Ray Chen, MD, ${ }^{\mathrm{b}}$ Chia-Jung Wang, MLS, Shun-Ying Yin, MD, ${ }^{\mathrm{e}}$ Shen-Hao Lai, MD, ${ }^{\mathrm{d}}$ and Kin-Sun Wong, $\mathrm{MD}^{\mathrm{d}}$

\section{ABSTRACT}

Objective: To determine gene expression profiles associated with bullae formation in patients with primary spontaneous pneumothorax and to identify candidate genes associated with surgical intervention.

Methods: Twenty-four adolescents with primary spontaneous pneumothorax were enrolled prospectively. A global gene expression analysis of 9 paired lung biopsies (lesion and normal adjacent sites) was performed to identify differentially expressed genes associated with spontaneous pneumothorax. Pathway and network analysis was performed using the Database for Annotation, Visualization and Integrated Discovery web tool. Candidate genes and encoding proteins were assessed in blood samples and compared between patients with pneumothorax and healthy control patients.

Results: A total of 1519 differentially expressed transcripts corresponding to known genes were identified comparing the lesion lung with paired adjacent normal lung. The altered genes were mainly associated with focal adhesion and extracellular matrix-receptor interaction pathways. Genes involved in proteolysis and peptidase activity were up-regulated predominantly, especially matrix metalloproteinase- 1 and -9 genes. Compared with the recovery stage, blood levels of matrix metalloproteinase-9/tissue inhibitor of metalloproteinase- 1 were increased at the acute stage in patients with pneumothorax and, when compared between patients treated operatively with those treated nonoperatively, were also significantly greater. In addition, ratios of their serum levels were significantly greater in patients with pneumothorax compared with healthy control patients. Furthermore, matrix metalloproteinase- 9 was

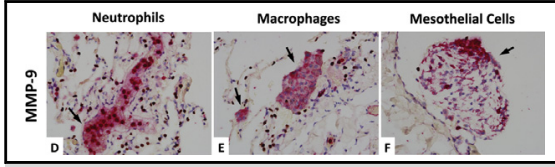

Overexpression of matrix metalloproteinase-9 immunostaining in the cells of lung tissues.

\section{Central Message}

An imbalance of cell-extracellular matrix interactions with overexpressed matrix metalloproteinases appears to be associated with primary spontaneous pneumothorax in adolescents.

\section{Perspective}

In adolescents with primary spontaneous pneumothorax, gene network and pathway analysis reveal up-regulated biologic processes of proteolysis and peptidase associated with lung tissues with blebs or bullae. Overexpression of matrix metalloproteinase- 9 may particularly play a role in contributing to the susceptibility of the bullae rupture with spontaneous pneumothorax.

See Editorial Commentary page 2337. predominantly overexpressed in neutrophils, alveolar macrophages, and mesothelial cells of lung biopsies.

Conclusions: An imbalance of cell-extracellular matrix interactions appears to be associated with primary spontaneous pneumothorax. Matrix metalloproteinase-9 overexpression may particularly play a role in contributing to pleural porosity for surgical intervention. (J Thorac Cardiovasc Surg 2018;156:2328-36)

From the Departments of ${ }^{\mathrm{a} P e d i a t r i c s}$ and ${ }^{\mathrm{b}}$ Pathology, Chang Gung Memorial Hospital at Keelung, College of Medicine, Chang Gung University, Taoyuan, Taiwan; ${ }^{\mathrm{c}}$ Graduate Institute of Clinical Medical Sciences, College of Medicine, Chang Gung University, Taoyuan, Taiwan; ${ }^{\mathrm{d}}$ Division of Pediatric Pulmonology, Department of Pediatrics, Chang Gung Memorial Hospital at Linkou, College of Medicine, Chang Gung University, Taoyuan, Taiwan; and ${ }^{\mathrm{e}}$ Department of Thoracic $\&$ Cardiovascular Surgery, Chang Gung Memorial Hospital at Keelung, Keelung City, Taiwan.

This work was supported by CMRPG2D0331-2 and CMRPG2B0391-2 from the Chang Gung Medical Foundation, Chang Gung University, Taiwan.

Drs Chen and Wong contributed equally to this article.
Received for publication June 24, 2017; revisions received May 6, 2018; accepted for publication May 9, 2018; available ahead of print July 19, 2018.

Address for reprints: Tzu-Ping Chen, MD, Department of Thoracic \& Cardiovascular Surgery, Chang Gung Memorial Hospital at Keelung, Taiwan, No. 222, Mai-chin Rd, Keelung, Taiwan (E-mail: kk13490@yahoo.com.tw); or Kin-Sun Wong, MD, Division of Pediatric Pulmonology, Chang Gung Memorial Hospital, College of Medicine, Chang Gung University, Taoyuan, Taiwan, No 5, Fu-Hsin St, Kueishan, Taoyuan, Taiwan (E-mail: pchest@adm.cgmh.org.tw). $0022-5223 / \$ 36.00$

Copyright (c) 2018 by The American Association for Thoracic Surgery https://doi.org/10.1016/j.jtcvs.2018.05.083 

Abbreviations and Acronyms
BMI = body mass index
$\mathrm{ECM}=$ extracellular matrix
IL $\quad=$ interleukin $\begin{aligned} \mathrm{KEGG}= & \text { Kyoto Encyclopedia of Genes and } \\ & \text { Genomes }\end{aligned}$
MMP = matrix metalloproteinase
$\mathrm{PBMC}=$ peripheral blood mononuclear cell
$\mathrm{PCR}=$ polymerase chain reaction
PSP = primary spontaneous pneumothorax
TIMP $=$ tissue inhibitor of metalloproteinase
TTF-1 $=$ thyroid transcription factor 1
VATS $=$ video-assisted thoracoscopic surgery

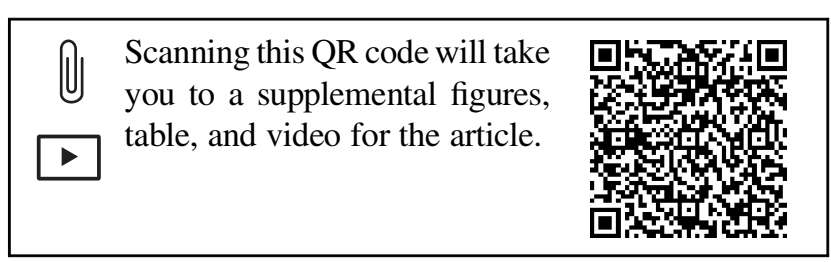

Primary spontaneous pneumothorax (PSP), which is defined as a pneumothorax without apparent lung disease, is a common clinical problem. ${ }^{1}$ PSP predominantly occurs at rest and in tall, thin male patients with a low body mass index (BMI). ${ }^{2}$ Although cigarette smoking is clearly implicated in the development of initial pneumothorax, the low prevalence of smoking in adolescent PSP implies the occurrence of PSP may be smoke-related but not crucial. ${ }^{3,4}$ PSP is potentially life-threatening, and its recurrence is always a serious problem. Despite the risk factors associated with PSP, a detailed understanding of the pathogenesis of PSP will likely provide much-needed clinical insights.

The occurrence of PSP is believed to result mainly from the spontaneous rupture of a subpleural bleb or of a bullae. ${ }^{5}$ The development of blebs or bulla might be related to many factors, such as distal airway inflammation, distal bronchial tree anomaly, apical ischemia, or abnormal connective tissue. ${ }^{6}$ PSP is reportedly caused by areas of disrupted mesothelial cells at the visceral pleura, replaced by an inflammatory elastofibrotic layer with increased porosity, which are prone to rupture. ${ }^{6}$ However, the mechanism of bulla formation and the pathogenesis of the spontaneous occurrence of a communication between the alveolar space and the pleura remain unknown.

Microarray technologies have risen to a robust and widely accepted tool in studying the pathogenesis of pulmonary diseases. ${ }^{7}$ A recent microarray study of PSP identified a number of hypoxia-, apoptosis-, and inflammation-related genes that may potentially induce the apoptosis of bronchoepithelial, alveolar, and mesothelial cells, resulting in the damage of alveolar sac and interstitial pleural lining structure and subsequent formation of blebs and bulla. However, these differentially expressed genes were obtained from a comparison of lung tissues from a wide age range of patients with different diseases, which may not fully represent the mechanism of bulla formation for PSP.

The aim of this study was to determine potential pathways involved in mediating the pathogenesis of PSP using microarray analysis of differential gene expression profiles in the lesion and normal adjacent sites of lung tissues from patients with PSP. Furthermore, we also sought to determine whether there was an association between expression of candidate genes and surgical intervention for the management of PSP.

\section{METHODS}

\section{Study Population and Design}

Patients with PSP, defined by spontaneous air accumulation in the pleural cavity without apparent underlying lung disease or trauma, were enrolled prospectively from August 2014 to December 2016. Patients with PSP were stratified into 2 groups: with and without surgery. Resection of PSP by video-assisted thoracoscopic surgery (VATS) was considered if patients had recurrent ipsilateral pneumothorax, prolonged air leak over 4 days in the first episode of pneumothorax, and large size of pneumothorax with or without tension pneumothorax. ${ }^{4,9,10}$ Lung tissue samples were obtained from patients who underwent VATS with wedge resection of the lung (Video 1). Lung biopsies of lesion and normal adjacent sites were collected and paired from the same resected lung tissues. The lesion sites referred to lung tissues containing bullae. The normal adjacent sites of lung biopsies, used as comparative controls, were taken at least $3 \mathrm{~cm}$ from the bullae to avoid overlapping of diseased lung tissues. Global gene expression profiles of the lesion and normal adjacent sites of lung tissues from patients with PSP were assessed using oligonucleotide microarray and compared.

All blood samples were collected within 72 hours of admission in patients with clinical confirmation of the diagnosis of PSP (acute stage) and after 3 months from the total remission of the episode of pneumothorax (recovery stage). Healthy adolescents aged less than 20 years without a personal history of pneumothorax or other pulmonary conditions or infections

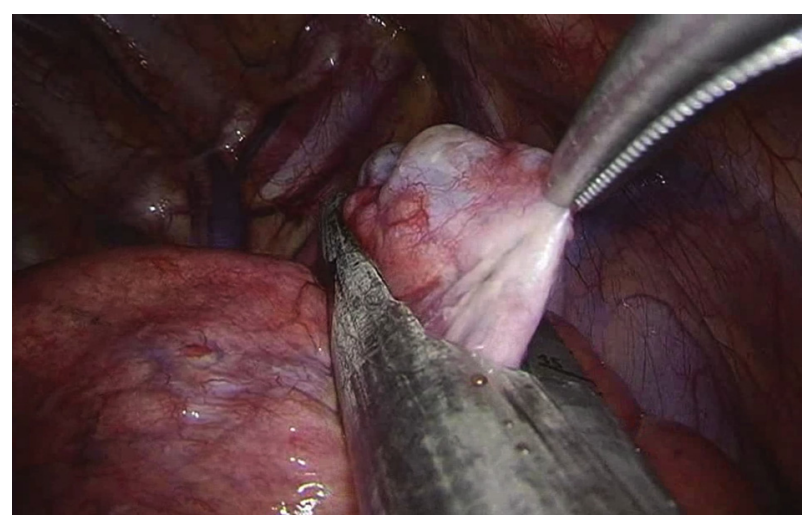

VIDEO 1. Demonstration of blebs or bullae at the apex of right upper lobe, followed by wedge resection using video-assisted thoracoscopic surgery. Video available at: https:/www.jtcvs.org/article/S0022-5223(18)31518-6/ fulltext. 
were enrolled, and their serum samples were collected as healthy controls. Candidate genes associated with PSP were determined in lung biopsies and compared in peripheral blood cells of patients with PSP between the acute and recovery stage and between with and without surgical intervention. Encoding proteins of PSP candidate genes were examined in serum and compared with healthy control patients and each other. Protein expressions were also assessed in lung tissues of patients with PSP undergoing VATS (Figure E1).

The details of demographic and clinical information, including age, sex, BMI, smoking habits, location and initial size of pneumothorax, treatment modalities, and length of hospital stay were recorded and analyzed. This study was approved by the institutional review board of Chang Gung Memorial Hospital (No. 103-6472C). All experiments in this study were performed in accordance with the relevant guidelines, and regulations and written informed consent was obtained from the parents of all patients with PSP and healthy control patients.

\section{RNA Isolation from Peripheral Blood Mononuclear Cells (PBMCs)}

PBMCs were isolated by centrifugation at $805 \times \mathrm{g}$ for 15 minutes (Eppendorf centrifuge 5810R; Thermo Fisher Scientific, Waltham, Mass) in Ficoll-Paque PLUS density gradient media (GE Healthcare BioSciences, Pittsburgh, Pa). Total RNA was isolated from PBMC with the RNeasy mini kit (QIAGEN, Valencia, Calif) following the manufacturer's instructions. Extracted RNA was then stored at $-80^{\circ} \mathrm{C}$ until further use.

\section{RNA Isolation From Lung Tissues}

Approximately 50 to $100 \mathrm{mg}$ of frozen lung tissues from liquid nitrogen were homogenized by using a mortar and pestle, and total RNA was extracted from the supernatant using the TRIzol reagent (Invitrogen/Thermo Fisher Scientific) according to manufacturer's instructions. The quality of the RNA samples was assessed using the RNA 6000 Nano Assay kit with the Agilent 2100 Bioanalyzer (Agilent Technologies, Santa Clara, Calif). An RNA integrity number was generated for each Bioanalyzer trace using 2100 Expert Software (Agilent Technologies). High-quality RNA, with RIN greater than 7 and A260/280 greater than 1.8, was considered for subsequent microarray experiments.

\section{Gene Expression Profiling Using Microarray}

A total of $0.2 \mu \mathrm{g}$ of RNA was amplified by a Low Input Quick-Amp Labeling kit (Agilent Technologies) and labeled with Cy3 (lesion sites) or Cy5 (normal adjacent sites) (CyDye; Agilent Technologies) during the in vitro transcription process. Correspondingly fragmented labeled cRNA was pooled and hybridized to an Agilent SurePrint G3 Human GE $8 \times 60 \mathrm{~K}$ Microarray (Agilent Technologies) at $65^{\circ} \mathrm{C}$ for 17 hours. Microarrays were scanned with an Agilent microarray scanner (Agilent Technologies) at $535 \mathrm{~nm}$ for $\mathrm{Cy} 3$ and $625 \mathrm{~nm}$ for Cy5. Scanned images were analyzed by Feature extraction 10.5.1.1 software (Agilent Technologies) to quantify the signal and background intensity and normalize the data using the rank-consistency-filtering LOWESS method. Statistical analysis of gene expression differences was performed using paired $t$ test with a false discovery rate of $1 \%$. Genes with significant differential expression were filtered using the cut-off of fold change $\geq 2$ and false discovery rateadjusted $P$ value $<.01$ and were selected if they were found greater than or equal to two thirds of patients. ${ }^{11}$ Microarray profiling data have been deposited in Figshare (https://doi.org/10.6084/m9.figshare.4216521).

\section{Quantitative Real-Time Polymerase Chain Reaction (PCR)}

Reverse transcription was performed on $1 \mu \mathrm{g}$ of RNA, and cDNA was synthesized using the High-Capacity cDNA Reverse Transcription Kits (Applied Biosystems, Foster City, Calif) following manufacturer's instructions. Real-time PCR was performed using the Bio-Rad iQ5 detection system with SYBR Green mix (Bio-Rad Laboratories, Hercules, Calif). Primer pairs used were designed with the Primer 3 RT-PCR program and checked for sequence homology against known genes using the BLAST search program. ${ }^{12}$ Table E1 shows the details of primers used in this study. PCR cycling conditions of an initial activation step of $95^{\circ} \mathrm{C}$ for 10 minutes, followed by 45 cycles of denaturation at $95^{\circ} \mathrm{C}$ for $15 \mathrm{sec}-$ onds, annealing at $62^{\circ} \mathrm{C}$ for 25 seconds, and extension at $72^{\circ} \mathrm{C}$ for $10 \mathrm{sec}-$ onds. Calculation of the threshold value, standard curve preparation, and quantification of mRNA in the samples were performed using the iQ5 software provided by Bio-Rad.

\section{Enzyme-Linked Immunosorbent Assay}

Serum were isolated from whole blood by centrifugation at $1,811 \times \mathrm{g}$ for 10 minutes (Eppendorf centrifuge $5810 \mathrm{R}$ ) and stored at $-80^{\circ} \mathrm{C}$ until further use. Serum levels of matrix metalloproteinase (MMP)-1, MMP-9, tissue inhibitor of metalloproteinase (TIMP)-1, and interleukin (IL)-8 were measured with enzyme-linked immunosorbent assay kits (DuoSet; R\&D Systems; Minneapolis, Minn) according to the manufacturer's instructions.

\section{Lung Tissue Preparation and Immunohistochemistry}

Four micrometer-thick sections were used for immunohistochemical detection of target proteins (MMP-1 and MMP-9) in formalin-fixed, paraffin-embedded tissues. Thyroid transcription factor 1 (TTF-1) is a nuclear transcription factor that is found predominantly in normal type II alveolar pneumocytes. Double immunostaining of MMP-1, MMP-9, and TTF-1 was carried out using rabbit polyclonal antibody against human MMP-1 (1:100 dilution; Proteintech Group, Inc, Chicago, Ill), rabbit monoclonal antibody against human MMP-9 (1:100 dilution; Epitomics, Burlingame, Calif), and mouse monoclonal antibody against TTF-1 (1:200 dilution; Leica Microsystems, Melbourne, Australia). Pretreatment of sections (deparaffinization, rehydration, and antigen retrieval) and automated immunostaining were carried out in a BOND-MAX fully automated autostainer (Leica Microsystems). Each run of immunohistochemical staining contained a positive and a negative slide to ensure the quality of staining. Images were taken using the Olympus fluorescent microscope (Tokyo, Japan) equipped with DP71 CCD camera image capture system.

Each slide was evaluated in obvious lesions and was read and scored by 2 independent investigators. Staining intensity was scored semiquantitatively as follows: no staining (less than $10 \%$ of positive cells); weak staining (10\%-50\% of positive cells); and strong staining (more than $50 \%$ of cells staining positive). ${ }^{13}$

\section{Statistical Analysis}

The Student $t$ test was used to compare continuous variables, and the $\chi^{2}$ or Fisher exact test was used to compare the nominal data. Differences in continuous variables with non-normal distribution between PSP patients with, without VATS, and healthy control patients were tested using the Mann-Whitney $U$ test. Differences of candidate gene expression between paired lesion and normal adjacent biopsies and acute and recovery stages of patients with PSP were investigated using the nonparametric Wilcoxon signed-rank test. Statistical analysis was performed with the Statistical Package for the Social Sciences (SPSS Statistics for Windows Version 20.0, IBM Corp, Armonk, NY), and graphs were drawn using GraphPad Prism Version 5.01 software (GraphPad Software Inc, San Diego, Calif). All statistical hypothesis tests were 2-tailed, and a $P$ value $<.05$ was considered to be significant.

\section{RESULTS \\ Patient Characteristics}

A total of 47 subjects were enrolled into this study, including 24 patients with PSP and 23 healthy control 
TABLE 1. Clinical and radiographic differences between patients with PSP with and without VATS and with healthy control patients

\begin{tabular}{|c|c|c|c|c|c|c|}
\hline & Patients & PSP & & Patients with PSP & Control patients & \\
\hline & $\overline{\text { Non-VATS }(n=11)}$ & VATS $(n=13)$ & $P$ value & $\overline{\text { Total }(n=24)}$ & $\overline{\text { Healthy }(n=23)}$ & $P$ value \\
\hline Age, $y$ & $16.1 \pm 1.1$ & $18.1 \pm 1.0$ & $<.001$ & $17.2 \pm 1.4$ & $17.4 \pm 0.7$ & .464 \\
\hline Sex, male & $11(100 \%)$ & $13(100 \%)$ & & $24(100 \%)$ & $16(100 \%)$ & \\
\hline Body mass index & $18.1 \pm 1.3$ & $18.9 \pm 1.6$ & .218 & $18.5 \pm 1.5$ & $17.8 \pm 2.9$ & .323 \\
\hline Cigarette smoking & $2(18 \%)$ & $2(15 \%)$ & 1.000 & $4(17 \%)$ & $0(0 \%)$ & .089 \\
\hline $\begin{array}{l}\text { Episodes } \\
\text { First attack } \\
\text { Non-first attack }\end{array}$ & $\begin{array}{c}10(91 \%) \\
1(9 \%)\end{array}$ & $\begin{array}{l}5(38 \%) \\
8(62 \%)\end{array}$ & .013 & $\begin{array}{r}15(63 \%) \\
9(37 \%)\end{array}$ & & \\
\hline $\begin{array}{l}\text { Site } \\
\text { Right } \\
\text { Left }\end{array}$ & $\begin{array}{l}3(27 \%) \\
8(73 \%)\end{array}$ & $\begin{array}{l}5(38 \%) \\
8(62 \%)\end{array}$ & .679 & $\begin{array}{r}8(33 \%) \\
16(67 \%)\end{array}$ & & \\
\hline $\begin{array}{l}\text { Initial size } \\
\quad \text { Small } \\
\text { Medium } \\
\text { Large }\end{array}$ & $\begin{array}{l}7(64 \%) \\
4(36 \%) \\
0(0 \%)\end{array}$ & $\begin{array}{l}4(31 \%) \\
3(23 \%) \\
6(46 \%)\end{array}$ & .033 & $\begin{array}{r}11(46 \%) \\
7(29 \%) \\
6(25 \%)\end{array}$ & & \\
\hline $\begin{array}{l}\text { Treatment } \\
\text { Oxygen therapy only } \\
\text { Chest drains only }\end{array}$ & $\begin{array}{l}8(73 \%) \\
3(27 \%)\end{array}$ & - & & $\begin{array}{l}8(33 \%) \\
3(13 \%)\end{array}$ & & \\
\hline Hospital stay, d & $3.6 \pm 1.0$ & $7.2 \pm 2.7$ & $<.001$ & $5.5 \pm 2.8$ & & \\
\hline
\end{tabular}

Data shown are mean \pm SD or number $(\%)$ of patients as appropriate. Initial pneumothorax size is categorized as small, medium, or large depending on the size of the visible rim of air between the lung margin and the chest wall at level of hilum: small $(0 \mathrm{~cm})$; moderate $(<2 \mathrm{~cm})$; $\operatorname{large}(\geq 2 \mathrm{~cm}){ }^{4}$ The $P$ value is for comparison of patients with PSP between with and without VATS and between total patients with PSP and healthy controls respectively. PSP, Primary spontaneous pneumothorax; VATS, video-assisted thoracoscopic surgery.

patients (Table 1). All subjects were male, and there were no significant differences in age and BMI between healthy control patients and patients with PSP. Cigarette smoking was only found in 4 patients with PSP. Thirteen patients with PSP $(54 \%)$ underwent VATS. Non-first attack of PSP and a large-size pneumothorax were significantly different in patients with VATS in comparison with patients without surgical intervention. The average hospital stay (days) was longer in patients with PSP undergoing VATS than in those not receiving surgery $(7.2 \pm 2.7$ vs $3.6 \pm 1.0$, $P<.001)$.

\section{Analysis of Global Gene Expression in Paired Lung Biopsies of Patients With PSP}

To identify possible pathways involving in the development of PSP, gene expression profiles of lung tissues from patients with PSP receiving VATS were assessed and analyzed. A total of 10 paired lung biopsies (lesion and normal adjacent sites) were collected, and gene expression analysis experiments were performed in 9 paired lung biopsies that satisfied stringent criteria for microarray analysis. We found 1805 differentially expressed transcripts when we compared the diseased lung with paired adjacent normal lung; 1519 of the transcripts corresponded to known genes. Six hundred six up-regulated and 913 downregulated genes were identified and input into the Database for Annotation, Visualization and Integrated Discovery for
Kyoto Encyclopedia of Genes and Genomes (KEGG) pathway analysis and Gene Ontology enrichment analysis.

Genes that were up-regulated were involved in focal adhesion and extracellular matrix (ECM)-receptor interaction KEGG pathways. In addition to these pathways, downregulated genes were also involved in KEGG pathways tight junction and cell adhesion molecules (Table 2). Gene Ontology analysis of differentially expressed genes associated with PSP is shown in Figure 1. Enriched biologic terms related to proteolysis in biological process, ECM in cellular component, and peptidase, endopeptidase, and metallopeptidase activity in molecular function were up-regulated predominantly. Furthermore, MMPs genes including collagenases (MMP-1 and $M M P-13)$, gelatinases $(M M P-2$ and $M M P-9)$, stromelysins $(M M P-3$ and $M M P-11)$, membrane-type (MMP-14 and $M M P-16)$, matrilysin (MMP-7), and TIMP-1 and TIMP-4 involved in the molecular functions of peptidase activity were significantly overexpressed (Figure 2). Among them, $M M P-1$ and $M M P-9$ expressed with more than 10 -fold difference were selected for microarray data validation and studied further.

\section{$M M P-1, M M P-9$, and $I L-8$ Gene Expression in Lung Biopsies and PBMCs}

Balance in the MMPs activity is usually achieved by TIMPs, and IL-8 is known to up-regulate MMP-9 production. ${ }^{14}$ To determine the association of PSP candidate genes 
TABLE 2. KEGG pathways significantly enriched within genes differentially expressed between lesion and normal adjacent sites of lung biopsies in patients with primary spontaneous pneumothorax

\begin{tabular}{|c|c|c|c|c|c|}
\hline KEGG pathway & Count & $\%$ & $\mathbf{F E}$ & $P$ value & Benjamini \\
\hline \multicolumn{6}{|l|}{ From genes up-regulated } \\
\hline Neuroactive ligand-receptor interaction & 18 & 0.32 & 2.02 & $6.94 \mathrm{E}-03$ & 0.207 \\
\hline Focal adhesion & 16 & 0.29 & 2.29 & $3.82 \mathrm{E}-03$ & 0.156 \\
\hline ECM-receptor interaction & 15 & 0.27 & 5.13 & 8.07E-07 & $<0.001$ \\
\hline Complement and coagulation cascades & 9 & 0.16 & 3.75 & $2.42 \mathrm{E}-03$ & 0.149 \\
\hline \multicolumn{6}{|l|}{ From genes down-regulated } \\
\hline Pathways in cancer & 28 & 0.37 & 1.72 & $5.74 \mathrm{E}-03$ & 0.098 \\
\hline Focal adhesion & 20 & 0.26 & 1.20 & 4.70E-03 & 0.107 \\
\hline Endocytosis & 19 & 0.25 & 2.08 & 4.10E-03 & 0.112 \\
\hline Tight junction & 17 & 0.22 & 2.55 & 8.60E-04 & 0.040 \\
\hline Cell adhesion molecules & 16 & 0.21 & 2.44 & 2.06E-03 & 0.071 \\
\hline ECM-receptor interaction & 14 & 0.18 & 3.35 & 2.13E-04 & 0.030 \\
\hline Complement and coagulation cascades & 12 & 0.16 & 3.50 & $5.02 \mathrm{E}-04$ & 0.035 \\
\hline Dilated cardiomyopathy & 12 & 0.16 & 2.62 & $5.40 \mathrm{E}-03$ & 0.105 \\
\hline Hypertrophic cardiomyopathy & 11 & 0.14 & 2.60 & $8.78 \mathrm{E}-03$ & 0.119 \\
\hline Hedgehog signaling pathway & 9 & 0.12 & 3.23 & $5.85 \mathrm{E}-03$ & 0.090 \\
\hline
\end{tabular}

Pathways were identified by the Database for Annotation, Visualization and Integrated Discovery Functional Annotation and selected by using a $P$ value $<.01$. Counts and percentages refer to the number and percentage of genes from the input list that fit into a given KEGG pathway. KEGG, Kyoto Encyclopedia of Genes and Genomes; $F E$, fold enrichment is the magnitude of enrichment for each KEGG pathway compared with the entire human genome that serves as the reference; ECM, extracellular matrix.

in peripheral blood system to lung tissues and surgical intervention, $M M P-1, M M P-9$, and $I L-8$ genes were analyzed in lung biopsies and PBMCs of patients with PSP. Gene expression ratios of $M M P-1 / T I M P-1$ and $M M P-9 / T I M P-1$ were determined in lesion sites from lung biopsy tissue of patients with PSP and found to be significantly greater
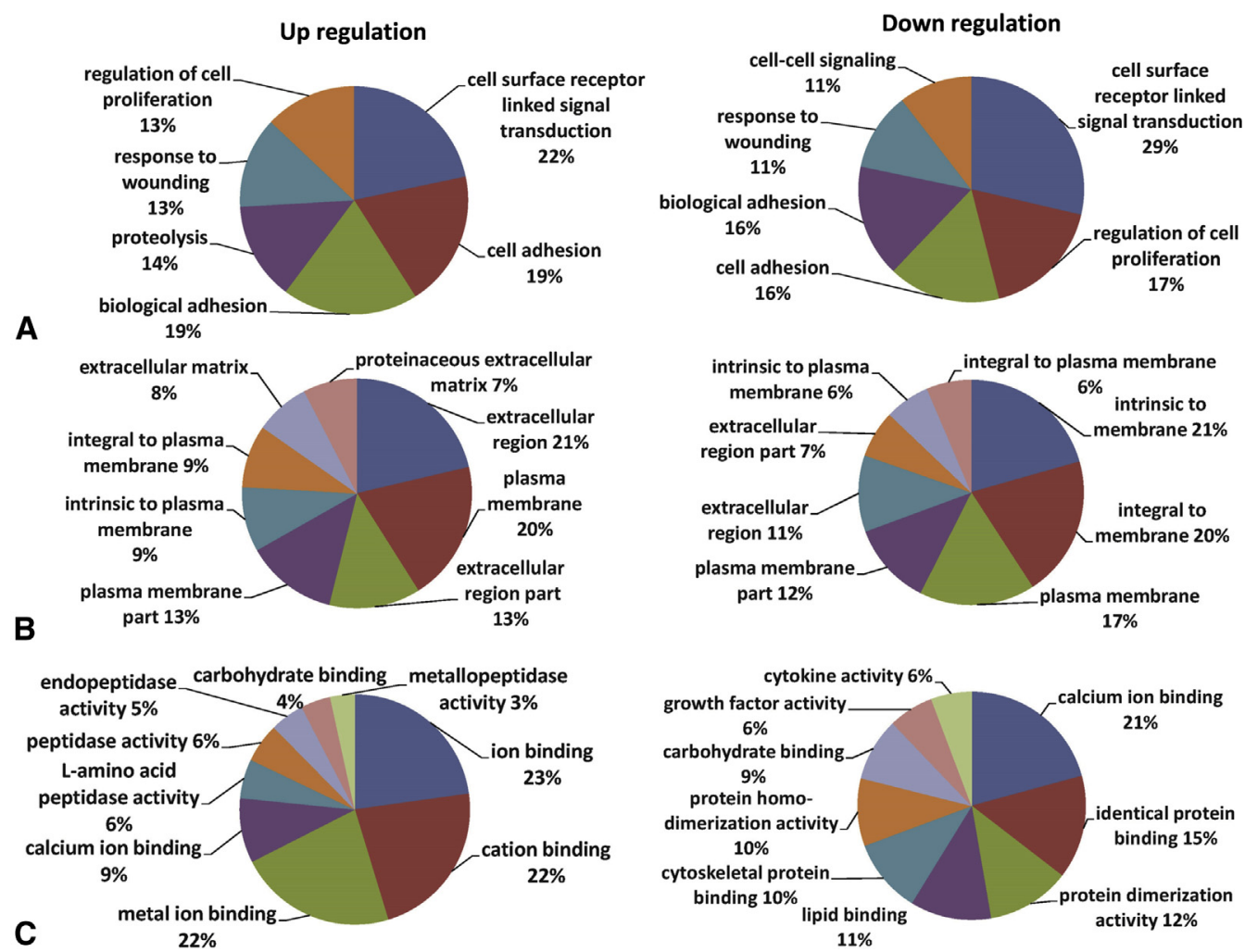

FIGURE 1. Enrichment of Gene Ontology terms in differentially expressed up- and down-regulated genes associated with PSP. The enriched Gene Ontology terms according to (A) biological process, (B) cellular component, and (C) molecular function. 


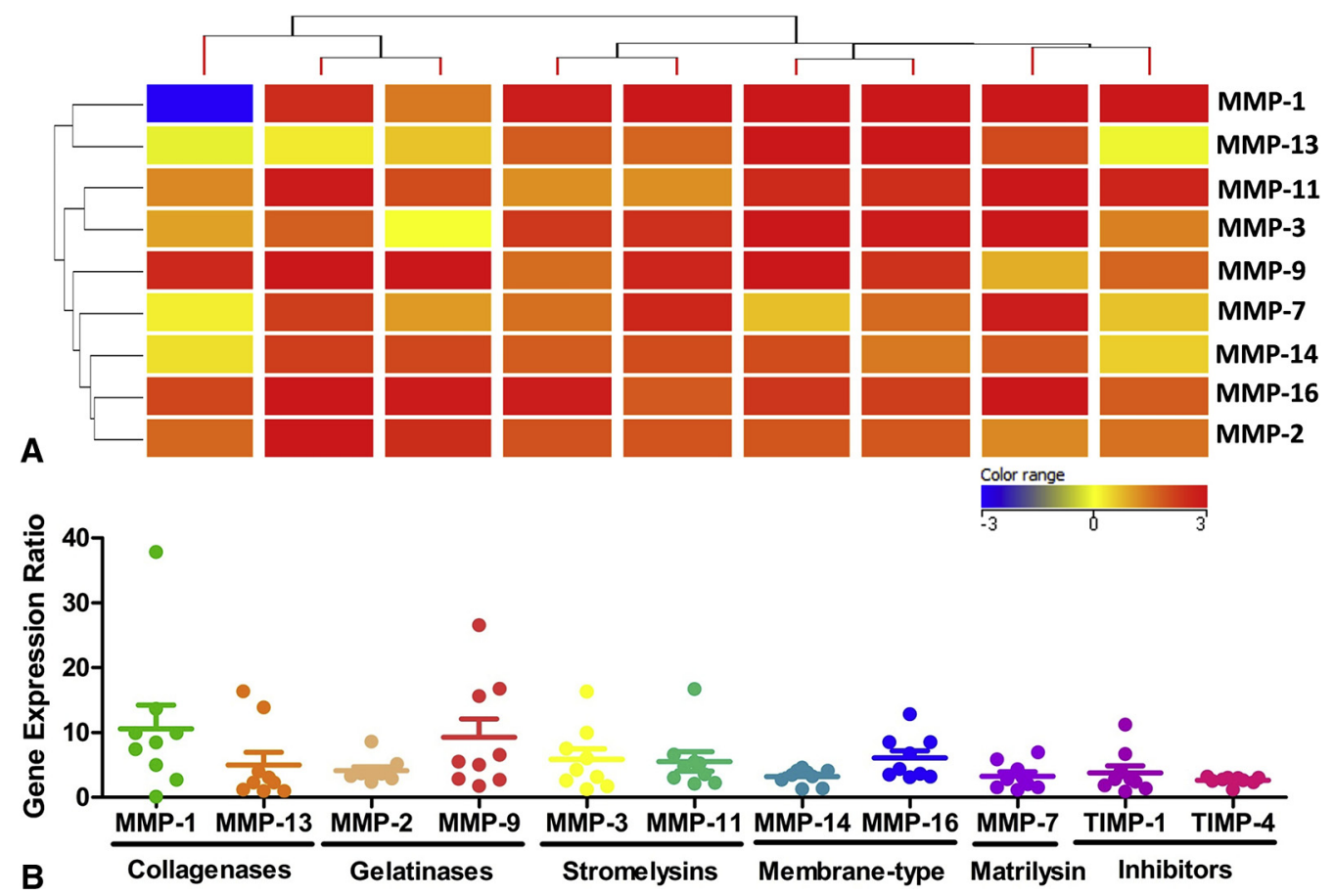

FIGURE 2. MMP genes differentially expressed in lung tissues of 9 patients with PSP. Hierarchical clustering of 9 MMP gene expression profiles across 9 patients (A) and gene expression ratios of paired lesion and normal site tissues plotted from the microarray data (B). Red or blue colors indicate higher and lower expression levels, respectively, of the genes correlating into a smaller number of uncorrelated variables called principal components. MMP, Matrix metalloproteinase; TIMP, tissue inhibitor of metalloproteinase.

compared with paired adjacent normal lung tissue (Figure 3, $A)$. In contrast, when we compared $M M P-1 / T I M P-1$, $M M P-9 / T I M P-1$ ratios and $I L-8$ in the PBMCs of patients with PSP in the acute versus recovery stages, $M M P-9 /$ TIMP- 1 and $I L-8$ but not MMP-1/TIMP- 1 were significantly greater (Figure 3, B). Furthermore, MMP-9/TIMP-1 gene expression ratio in PBMCs during the acute stage was significantly greater in patients with PSP who underwent VATS compared with those managed without surgery (Figure 3, C). In addition to PBMCs, we also examined the expression of PSP candidate genes in blood serum. In contrast to PBMCs, there was not a statistically significant difference in serum levels of MMP-9/TIMP-1 ratio between the acute and recovery stage for all patients with PSP. However, compared with healthy control patients, the ratio of serum levels of MMP-9/TIMP-1 were significantly greater in patients with PSP at the acute and recovery stage (Figure 3,D).

\section{MMP-1 and MMP-9 Expression in Lung Tissues by Immunohistochemistry}

To identify cellular and molecular mechanisms of MMPs involving in PSP, protein expressions of MMP-1 and MMP9 in the lung biopsies of patients with PSP receiving VATS $(\mathrm{n}=13)$ were assessed by immunohistochemistry. Alveolar type II cells displayed strong TTF-1 nuclear staining without obvious MMP-1 and MMP-9 expression due to high nuclear/cytoplasmic ratio. Bronchiolar epithelial cells displayed strong TTF-1 nuclear staining with obvious MMP-1 expression but not that of MMP-9. MMP-1 was relatively overexpressed in the macrophages and mesothelial cells, but MMP-9 was highly overexpressed in the neutrophils, macrophages, and mesothelial cells of PSP lung specimens (Figure 4). Figure E2 illustrates another 12 patients with PSP showing strong staining of MMP-9 in neutrophils (12 patients), in macrophages (10 patients), and in mesothelial cells (8 patients).

\section{DISCUSSION}

PSP represents a common clinical problem and can potentially be life-threatening. ${ }^{6}$ The exact pathogenesis of blebs or bullae formation and rupture at the apices of the lungs remains unclear. Microarray technologies potentially offer an opportunity to provide new insights into the pathogenesis of PSP. This study has demonstrated an imbalance of cell-ECM interactions associated with PSP in adolescents. Overexpression of MMPs with increased biologic process of proteolysis may contribute to susceptibility of the tissues to risk of rupture.

Pathway-based microarray analysis allows a comprehensive understanding of the molecular mechanisms underlying complex diseases. ${ }^{15}$ In this study, differential 
Lung Biopsies of PSP Patients

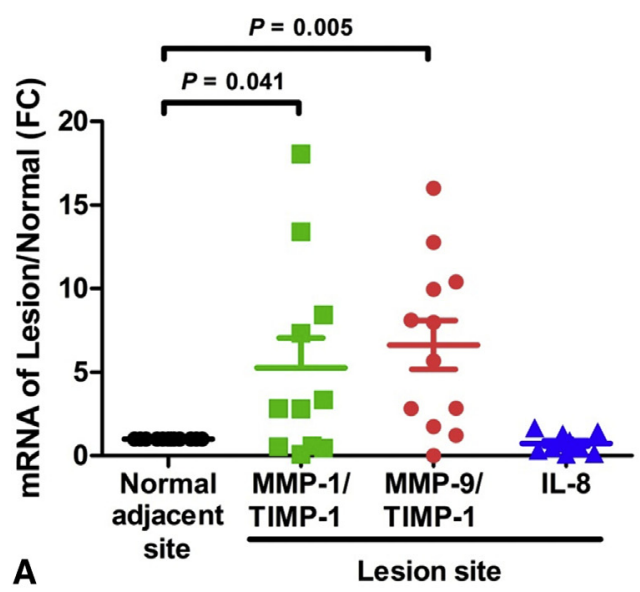

PBMCs of PSP Patients

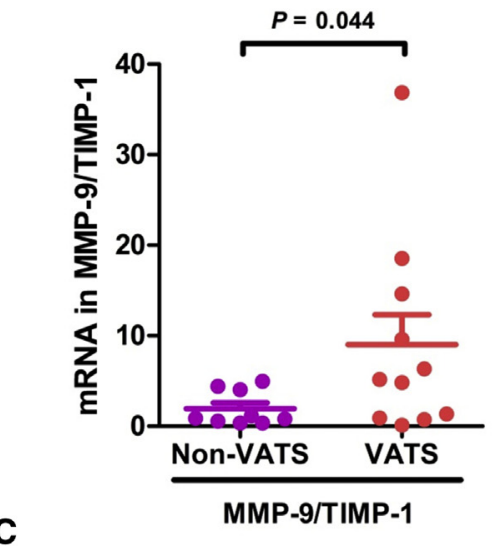

PBMCs of PSP Patients

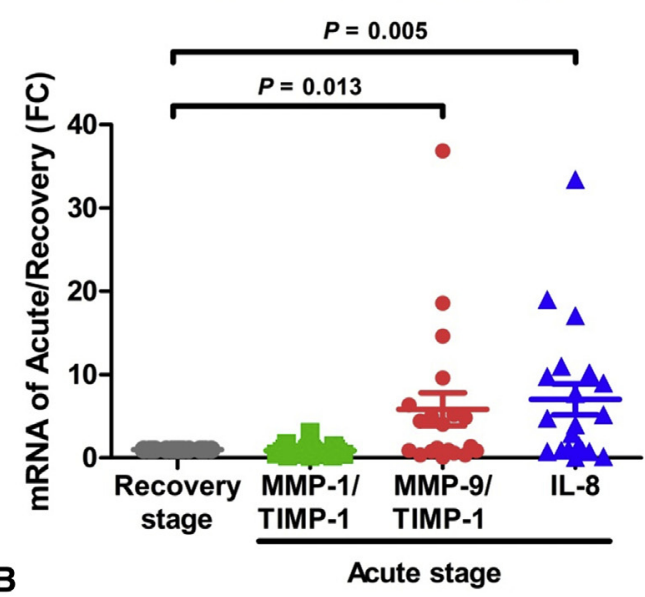

Serum of PSP Patients and Controls

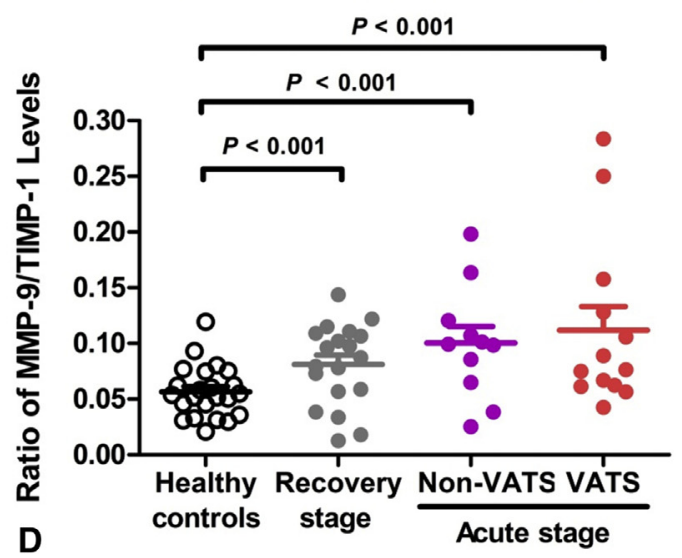

FIGURE 3. Fold changes of $M M P-1 / T I M P-1, M M P-9 / T I M P-1$, and $I L-8$ gene expression in lung tissues (A) and PBMCs (B). Comparisons and differences of $M M P-9 / T I M P-1$ gene expression ratios between patients with and without VATS (C), and of ratios of serum $M M P-9 / T I M P-1$ levels between healthy control patients and patients with PSP at acute and recovery stage (D). PSP, Primary spontaneous pneumothorax; $F C$, fold changes; $M M P$, matrix metalloproteinase; TIMP, tissue inhibitor of metalloproteinase; $I L$, interleukin; PBMC, peripheral blood mononuclear cell; VATS, video-assisted thoracoscopic surgery.

expressions of genes involved in PSP were found to be associated with pathways related to focal adhesion and ECMreceptor interactions in the lung. At the cell-ECM contact points, specialized structures are formed and termed focal adhesions, where transmembrane adhesion receptors provide a structural link between the actin cytoskeleton and the ECM components. ${ }^{16}$ In addition, cell adhesion molecules are proteins expressed on the cell surface and play an important physical role in shaping the structure of multicellular organisms. ${ }^{17}$ Our results suggest that a breakdown of cell-ECM interactions in the lung may potentially contribute to the formation of bullae in adolescents with PSP.

Biological analysis using Gene Ontology provides a technique that allows a global overview of the functional annotations that are significantly enriched in a list of genes. ${ }^{18} \mathrm{In}$ this study, biologic functions including proteolysis, peptidase, and metallopeptidase were significantly upregulated in bullous lung biopsies of patients with PSP. Genes of MMPs, zinc-dependent endopeptidases belonging to a larger family of proteases, were significantly enriched in the up-regulated genes. MMPs are produced from inflammatory and structural cells in the airways and play an important role in invasion of inflammatory cells by degrading the ECM. ${ }^{19}$ These findings suggest that increased MMPs expression associated with ECM degradation in the lung may particularly explain the mechanism of PSP development.

MMPs are classified based on their substrate specificity into collagenases (MMP-1, -8, and -13), gelatinases (MMP-2 and -9), stromelysins (MMP-3, -7, and -10), elastases (MMP-12), and membrane-type MMPs. ${ }^{20,21}$ In this study, a number of these MMP genes, especially $M M P-1$ and $M M P-9$, were significantly differentially expressed in 


\section{Bronchiolar Epithelial Cells}

Macrophages

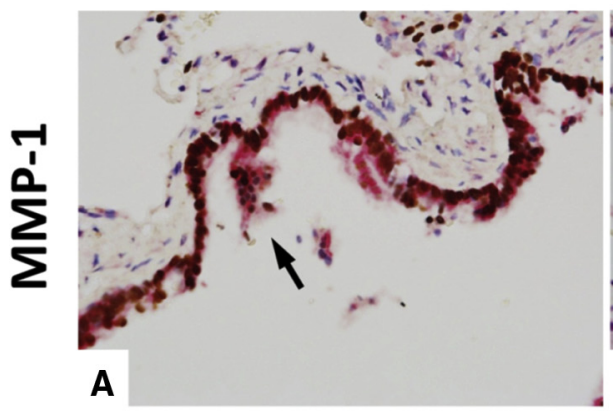

Neutrophils

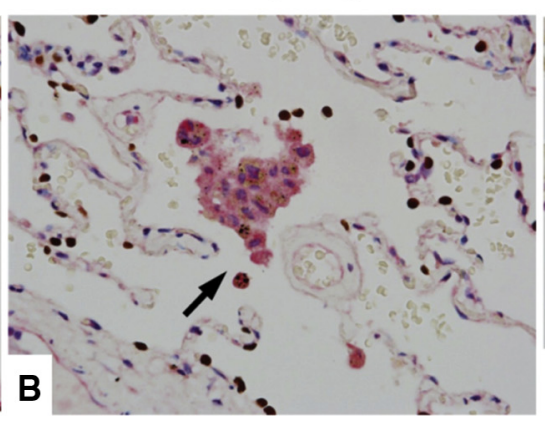

Macrophages
Mesothelial Cells

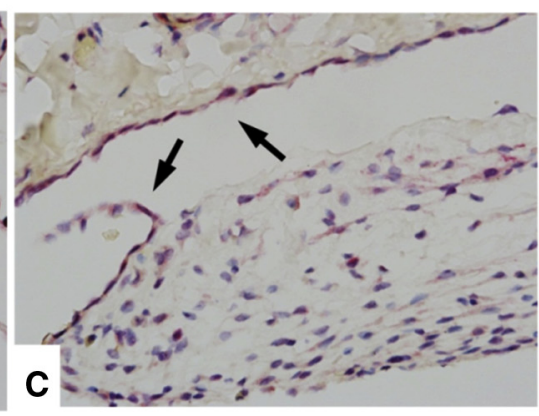

Mesothelial Cells
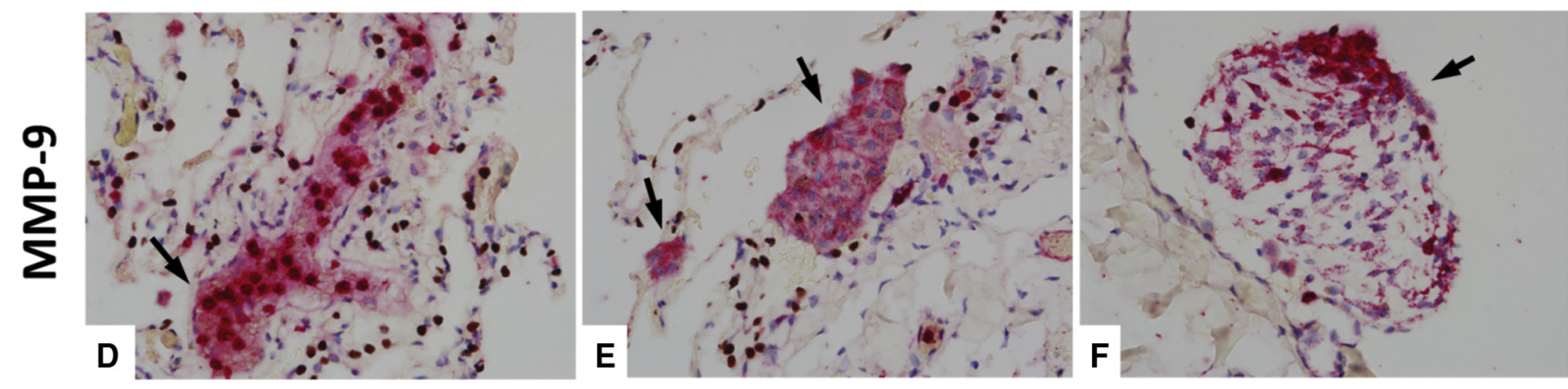

FIGURE 4. Representative micrographs $(\times 400)$ of MMP-1, MMP-9, and TTF-1 immunostaining in the lung specimens of patients with PSP. (A) Bronchiolar epithelial cells displayed strong TTF-1 nuclear staining (dark brown) with strong cytoplasmic MMP-1 immunoreactivity (red). (B) Aggregates of macrophages in the alveolar spaces were immunoreactive with MMP-1. (C) Mesothelial cells displayed hyperplasia with weak MMP-1 immunostaining. (D) Increased number of neutrophils (arrow) in the venules showed strong cytoplasmic MMP-9 immunoreactivity (red). Alveolar type II cells displayed strong TTF-1 nuclear staining (dark brown) without obvious MMP-9 expression. (E) Aggregates of macrophages (arrows) in the alveolar spaces were immunoreactive with MMP-9. (F) Mesothelial cells (arrow) displayed hyperplasia with strong MMP-9 immunostaining. MMP, Matrix metalloproteinase.

the pathologic lesions in the lungs of patients with PSP. MMP-1 and MMP-9 are important enzymes involved in the destruction of the lung and have been reported to be present in the lung parenchyma of patients with emphysema. ${ }^{22,23}$ Cellular and molecular mechanisms of MMPs in the lung may play an important role in the development of lung injury for PSP.

Recruited blood neutrophils and alveolar macrophages have been reported to be activated in chronic respiratory diseases with emphysema development. ${ }^{24,25}$ MMP-9 is secreted by neutrophils and macrophages and was predominantly stained in these cells in the pathologic lesions in the lungs of patients with PSP in this study. In addition, not only $M M P-9$ but also the $I L-8$ gene was found to be overexpressed in systemic circulation during at acute onset of PSP. IL-8, a chemotactic and inflammatory cytokine, is known to play a crucial role in the recruitment of neutrophils into the airways. However, the $I L-8$ gene was not expressed in PSP lung tissues without diffuse neutrophilic infiltrates, indicating that circulating IL-8 is likely in reaction to the PSP but not causing the PSP. In contrast, $M M P-9$ gene was overexpressed significantly in PSP lung tissues and systemic circulation, suggesting that circulating MMP-9 may provide a biologically relevant marker for PSP.
Mesothelial cells have multiple intercellular junctions and focal adhesions that anchor the mesothelial cell onto the ECM via integrins. ${ }^{26,27}$ Clinically, disrupted mesothelial cells at the visceral pleura are visible on thoracoscopy in patients with PSP. In this study, MMP-9 was not only overexpressed in the pleural mesothelial cells but also significantly expressed in patients with PSP who underwent surgery. Released MMP-9 within the mesothelial cells may play a crucial role in contributing to the imbalances between mesothelial cells and ECM with increased pleural porosity, allowing air leakage into pleural space and subsequent aggressive clinical intervention.

Limitations of our study include its small sample size for microarray gene expression profiling, with its high biological variability. In addition, because PSP commonly occurs in young adults, our use of healthy adolescents as controls may include individuals who subsequently develop PSP. However, a comprehensive tissue- and gene-specific analysis with a direct link between genes and proteins makes the results demonstrated here valid and potentially important.

In conclusion, microarray gene expression analysis provides a comprehensive review of the molecular pathogenesis of PSP. In patients with PSP, gene network and 
functional pathway analysis highlight the importance of an imbalance of cell-ECM interactions associated with blebs or bullae in the lung. Most importantly, biologic process of proteolysis and molecular function of peptidase and metallopeptidase are up-regulated in bullous lung biopsies. Overexpression of MMP-9 in the pleural mesothelial cells and alveolar macrophages may particularly play a role in contributing to susceptibility of the bullae rupture for surgical intervention. However, further studies are needed to translate this association into a cause-effect relationship.

\section{Conflict of Interest Statement}

Authors have nothing to disclose with regard to commercial support.

We express our gratitude to Dr Tsan-Yu Hsieh for providing expert assistance in pathology analysis. We are also extremely grateful to all the families who took part in this study and all pediatricians for their help in recruiting them.

\section{References}

1. Wallach SL. Spontaneous pneumothorax. N Engl J Med. 2000;343:300, author reply -1 .

2. Gupta D, Hansell A, Nichols T, Duong T, Ayres JG, Strachan D. Epidemiology of pneumothorax in England. Thorax. 2000;55:666-71.

3. Cheng YL, Huang TW, Lin CK, Lee SC, Tzao C, Chen JC, et al. The impact of smoking in primary spontaneous pneumothorax. J Thorac Cardiovasc Surg. 2009;138:192-5.

4. Chiu CY, Chen TP, Wang CJ, Tsai MH, Wong KS. Factors associated with proceeding to surgical intervention and recurrence of primary spontaneous pneumothorax in adolescent patients. Eur J Pediatr. 2014;173:1483-90.

5. Tschopp JM, Bintcliffe O, Astoul P, Canalis E, Driesen P, Janssen J, et al. ERS task force statement: diagnosis and treatment of primary spontaneous pneumothorax. Eur Respir J. 2015;46:321-35.

6. Noppen M. Spontaneous pneumothorax: epidemiology, pathophysiology and cause. Eur Respir Rev. 2010;19:217-9.

7. Campbell JD, Spira A, Lenburg ME. Applying gene expression microarrays to pulmonary disease. Respirology. 2011;16:407-18.

8. Fang HY, Lin CY, Chow KC, Huang HC, Ko WJ. Microarray detection of gene overexpression in primary spontaneous pneumothorax. Exp Lung Res. 2010;36: 323-30.

9. Choi SY, Kim YH, Jo KH, Kim CK, Park JK, Cho DG, et al. Video-assisted thoracoscopic surgery for primary spontaneous pneumothorax in children. Pediatr Surg Int. 2013;29:505-9.
10. Hsin MK. Another innovation in surgery for primary spontaneous pneumothorax (for men only). J Thorac Cardiovasc Surg. 2016;152:1006-7.

11. Dalman MR, Deeter A, Nimishakavi G, Duan ZH. Fold change and p-value cutoffs significantly alter microarray interpretations. BMC Bioinformatics. 2012; 13(suppl 2):S11.

12. Altschul SF, Madden TL, Schaffer AA, Zhang J, Zhang Z, Miller W, et al. Gapped BLAST and PSI-BLAST: a new generation of protein database search programs. Nucleic Acids Res. 1997;25:3389-402.

13. Chen CK, Chen PR, Huang HC, Lin YS, Fang HY. Overexpression of matrix metalloproteinases in lung tissue of patients with primary spontaneous pneumothorax. Respiration. 2014;88:418-25.

14. Chakrabarti S, Patel KD. Regulation of matrix metalloproteinase-9 release from IL-8-stimulated human neutrophils. J Leukoc Biol. 2005;78:279-88.

15. Jin L, Zuo XY, Su WY, Zhao XL, Yuan MQ, Han LZ, et al. Pathway-based analysis tools for complex diseases: a review. Genomics Proteomics Bioinformatics. 2014;12:210-20.

16. Petit V, Thiery JP. Focal adhesions: structure and dynamics. Biol Cell. 2000;92: 477-94.

17. Lu P, Takai K, Weaver VM, Werb Z. Extracellular matrix degradation and remodeling in development and disease. Cold Spring Harb Perspect Biol. 2011;3.

18. Huntley RP, Sawford T, Martin MJ, O'Donovan C. Understanding how and why the Gene Ontology and its annotations evolve: the GO within UniProt. Gigascience. 2014;3:4.

19. Elkington PT, Friedland JS. Matrix metalloproteinases in destructive pulmonary pathology. Thorax. 2006;61:259-66.

20. Demedts IK, Brusselle GG, Bracke KR, Vermaelen KY, Pauwels RA. Matrix metalloproteinases in asthma and COPD. Curr Opin Pharmacol. 2005;5: 257-63.

21. Denlinger CE. Matrix metalloproteinases and bronchiolitis obliterans: wrapping the enigma in a riddle. J Thorac Cardiovasc Surg. 2015;149:1203-4.

22. Imai K, Dalal SS, Chen ES, Downey R, Schulman LL, Ginsburg M, et al. Human collagenase (matrix metalloproteinase-1) expression in the lungs of patients with emphysema. Am J Respir Crit Care Med. 2001;163:786-91.

23. Chaudhuri R, McSharry C, Spears M, Brady J, Grierson C, Messow CM, et al. Sputum matrix metalloproteinase-9 is associated with the degree of emphysema on computed tomography in COPD. Transl Respir Med. 2013;1:1-5.

24. Sharafkhaneh A, Hanania NA, Kim V. Pathogenesis of emphysema: from the bench to the bedside. Proc Am Thorac Soc. 2008;5:475-7.

25. Ishii T, Abboud RT, Wallace AM, English JC, Coxson HO, Finley RJ, et al. Alveolar macrophage proteinase/antiproteinase expression in lung function and emphysema. Eur Respir J. 2014;43:82-91.

26. Batra $\mathrm{H}$, Antony VB. Pleural mesothelial cells in pleural and lung diseases. $J$ Thorac Dis. 2015;7:964-80.

27. Lin JB, Chen JF, Lai FC, Li X, Qiu ML. Transareolar pulmonary bullectomy for primary spontaneous pneumothorax. J Thorac Cardiovasc Surg. 2016;152: 999-1005.

Key Words: adolescents, extracellular matrix, matrix metalloproteinase-9, primary spontaneous pneumothorax 


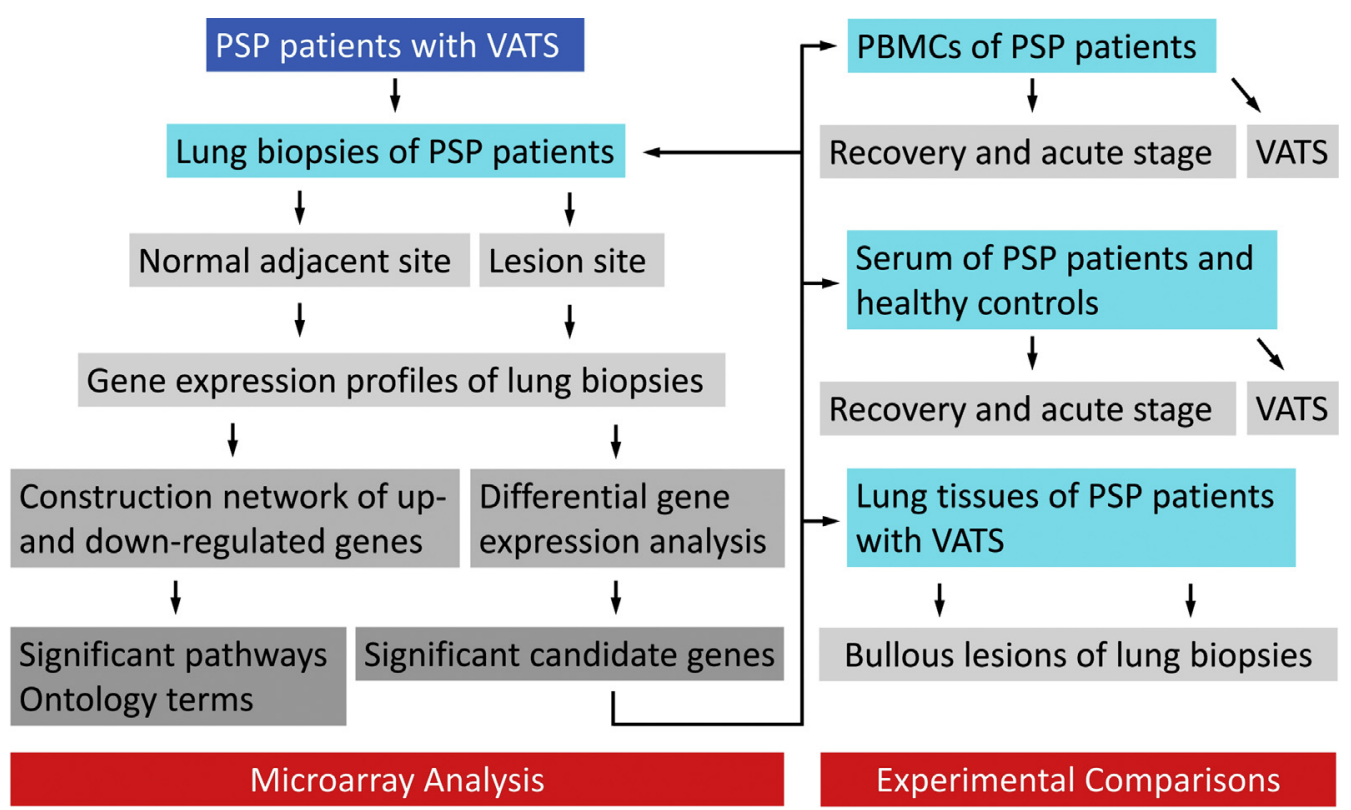

FIGURE E1. Flow chart of the overall microarray analysis and experimental outline. PSP, Primary spontaneous pneumothorax; VATS, video-assisted thoracoscopic surgery; PBMC, peripheral blood mononuclear cell. 


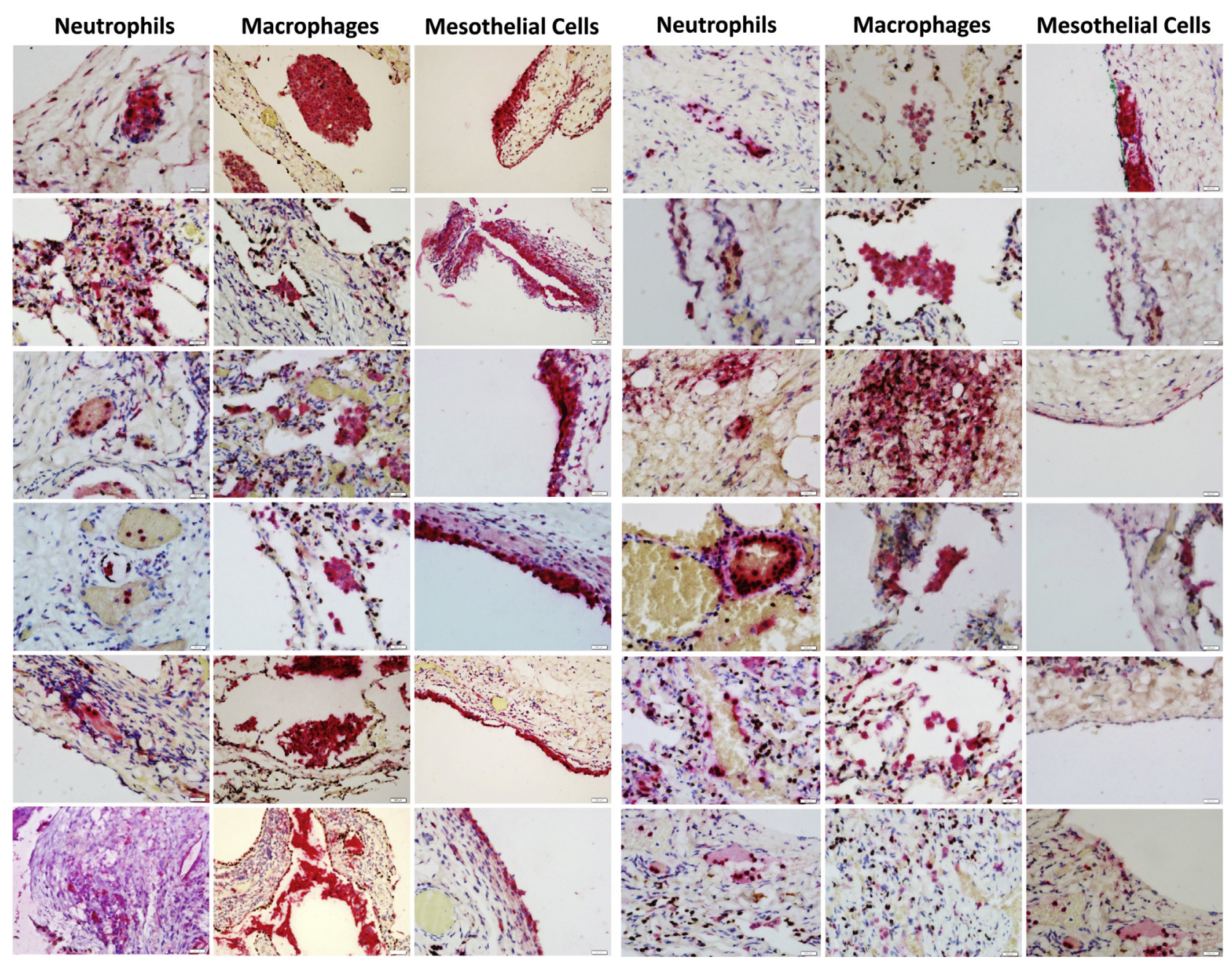

FIGURE E2. Representative micrographs $(\times 400)$ of MMP-9 and TTF-1 immunostaining in the lung specimens of patients with PSP. Neutrophils in the venules showed strong staining of MMP-9 but without neutrophil infiltration into interstitial tissues. Macrophages aggregated in the alveolar spaces with strong MMP-9 staining in 10 patients with PSP. Mesothelial cells displayed hyperplasia with strong MMP-9 staining in 8 patients with PSP.

TABLE E1. Primer sequences for target and housekeeping genes

\begin{tabular}{|c|c|c|}
\hline Gene & Primer sequence & PCR product size \\
\hline GAPDH & $\begin{array}{l}\text { Forward: 5'-TTCCAGGAGCGAGATCCCT-3' } \\
\text { Reverse: } 5^{\prime} \text {-CACCCATGACGAACATGGG-3' }\end{array}$ & 175 bp \\
\hline$M M P-1$ & $\begin{array}{l}\text { Forward: 5'-CAGAGATGAAGTCCGGTTTTTC- } 3^{\prime} \\
\text { Reverse: } 5^{\prime} \text {-GGGGTATCCGTGTAGCACAT- } 3^{\prime}\end{array}$ & 76 bp \\
\hline$M M P-9$ & $\begin{array}{l}\text { Forward: 5'-CCTGGAGACCTGAGAACCAATC-3' } \\
\text { Reverse: } 5^{\prime} \text {-CTCTGCCACCCGAGTGTAAC-3' }\end{array}$ & 84 bp \\
\hline TIMP-1 & $\begin{array}{l}\text { Forward: } 5^{\prime} \text {-GGGCTTCACCAAGACCTACA- } 3^{\prime} \\
\text { Reverse: } 5^{\prime} \text {-TGCAGGGGATGGATAAACAG-3' }\end{array}$ & $71 \mathrm{bp}$ \\
\hline$I L-8$ & $\begin{array}{l}\text { Forward: 5'-CTTGGCAGCCTTCCTGATTT-3' } \\
\text { Reverse: } 5^{\prime} \text {-TTCTTTAGCACTCCTTGGCAAAA-3' }\end{array}$ & $67 \mathrm{bp}$ \\
\hline
\end{tabular}

$\overline{P C R}$, Polymerase chain reaction; GAPDH, glyceraldehyde 3-phosphate dehydrogenase; MMP, matrix metalloproteinase; TIMP, tissue inhibitor of metalloproteinase; $I L$, interleukin. 